\title{
Propranolol for Treatment of Infantile Hemangioma: Efficacy and Effect on Pediatric Growth and Development
}

\author{
Rachel A. Giese $\mathbb{D}^{1},{ }^{1}$ Merit Turner $\mathbb{D}^{2},{ }^{2}$ Mario Cleves, ${ }^{3}$ J. Reed Gardner $\mathbb{D}^{2}{ }^{2}$ \\ and Gresham T. Richter $\oplus^{2}$ \\ ${ }^{1}$ Department of Surgery, Division of Otolaryngology-Head and Neck Surgery, University of Texas Rio Grande Valley School \\ of Medicine, 1201 W University Dr, Edinburg, TX, Canada 78539 \\ ${ }^{2}$ Department of Otolaryngology-Head and Neck Surgery, University of Arkansas of the Medical Sciences, 4301 W, St Little Rock, \\ Markham, AR, Canada 72205 \\ ${ }^{3}$ Morsani College of Medicine, Department of Pediatrics, Health Informatics Institute, University of South Florida, \\ 3650 Spectrum Blvd, Tampa, FL 33612, USA
}

Correspondence should be addressed to Gresham T. Richter; gtrichter@uams.edu

Received 18 January 2021; Accepted 10 March 2021; Published 8 April 2021

Academic Editor: Samuel Menahem

Copyright ( 2021 Rachel A. Giese et al. This is an open access article distributed under the Creative Commons Attribution License, which permits unrestricted use, distribution, and reproduction in any medium, provided the original work is properly cited.

\begin{abstract}
Purpose. Propranolol has been successful in treating problematic infantile hemangiomas (IH) but concerns regarding its effect on normal growth and development have been raised. This study examines physical growth, developmental milestones, and human growth hormone $(\mathrm{hGH})$ levels in infants receiving propranolol for problematic IH. Method. Monthly heights and weights of children undergoing propranolol therapy for IH were prospectively collected and tabulated. Data analysis and comparison to World Health Organization (WHO) weight-for-age and weight-to-length $z$-scores was performed. Questionnaires regarding milestones, efficacy, and guardian satisfaction were performed, and a combination of both chart results and phone conducted surveys was tabulated. Serum from a small representative cohort of age-matched children with IH treated and not treated with propranolol was collected. Results. A total of 185 children receiving propranolol therapy between 2008 and 2013 for IH were assigned to this study. The children were divided into two cohorts based on the presence of comorbidities or risk factors that may affect growth and development ( $n=142$ no comorbidities, $n=43$ with comorbidities). Neither cohort demonstrated deviation from normal weight in comparison to WHO normative data. There was a significant deviation for BMI-for-age and weight-for-age $z$-scores in our population, especially in patients on propranolol for more than 7 months. Based on data from participants, via either completed questionnaires or chart results, most children met their developmental milestones at or before target ages, regardless of the presence of comorbidities. Eighty percent of guardians noticed clinical improvement of the IH, with $91 \%$ either happy about or neutral to using the medication. hGH levels were higher in patients receiving propranolol therapy, but not significantly different. Conclusion. Propranolol therapy is effective and well tolerated in the treatment of infantile hemangiomas. This study suggests that propranolol does not impair growth and has no impact on normal pediatric development.
\end{abstract}

\section{Introduction}

Infantile hemangiomas $(\mathrm{IH})$ are benign tumors of blood vessels that, in general, start growing by the third month of life, undergo a rapid growth phase for 6-8 months, and subsequently involute at a variable rate. They are the most common benign tumor of infancy, occurring in about $5 \%$ of infants [1]. Most of them do not require treatment, but about
$12 \%$ are problematic and prompt referral to a specialist and require treatment [2]. IH characteristics that stimulate referral may include airway compromise, cosmetic deformity, ulceration, bleeding, or functional loss, such as visual impairment or dysphagia. In the past, steroids, chemotherapy, laser therapy, and surgery treatments were used for problematic $\mathrm{IH}$. These treatment options are becoming less necessary after the serendipitous discovery by Leaute-Labreze et al. 
[3] of early IH involution in a child treated with propranolol for cardiomyopathy. Since that discovery, physicians have embraced this therapy and are increasingly showing its success and safety.

Propranolol is a nonselective beta-blocker with a relatively low side effect profile, especially in healthy infants [4]. The most common side effects are sleep disturbance, dyspnea, nausea, somnolence, bradycardia, hypotension, hypoglycemia, and gastroesophageal reflux $[5,6]$. However, children treated with propranolol for IH are typically asymptomatic or experience mild hypotension or bradycardia [1]. Additionally, children with preexisting hypoglycemia have been safely treated for infantile hemangiomas with propranolol [7]. Reported deaths and instances of heart failure associated with use of propranolol have been due to intravenous propranolol or overdose [8]. Following FDA approval for the treatment of IHs and its increasing use in institutions around the world [3, 5, 9-17], the American Society of Pediatric Otolaryngology Vascular Anomalies Task Force approved its use $[1,2]$. Consensus conferences, international multicenter studies (NCT01056341), and meta-analysis have all shown the safety and efficacy of propranolol [18], especially in comparison to steroids [19]. Erbay et al. [20] demonstrated safety and efficacy in preterm and low birth weight infants. Some centers are successfully using a nonselective topical betablocker, such as timolol, with or without oral systemic propranolol to limit systemic toxicity $[21,22]$.

Although propranolol has been shown to have a clean side effect profile, questions regarding the effect of propranolol on normal growth and development have been raised among physicians treating children with IH. Pediatricians have questioned if potential long-term side effects of prolonged therapy on growth and development could be justified by the benefits for nonlife-threatening hemangiomas.

This study prospectively and longitudinally examines $1^{\text {st }}$ year growth parameters (height and weight) and developmental milestones up to 48 months of age in infants treated with oral propranolol on an outpatient basis. In addition, in a small cohort of treated patients, human growth hormone (hGH) levels were evaluated compared to age-matched normal controls. We hypothesize that propranolol does not influence normal pediatric growth and development. It is our hope that studies such as this will assuage these concerns and validate its use for children with problematic $\mathrm{IH}$.

\section{Methods}

Institutional Review Board approval 202187 and informed consent were obtained. First, a retrospective review of prospectively collected data on heights and weights of children on propranolol was performed. These values were recorded from monthly dosing changes based on weight as recorded by the patient's PCP.

Patients were divided into two cohorts, those with comorbidities that could affect growth and development and those without such comorbidities. The most common reason a patient was determined to have a comorbidity was a gestational age less than 36 weeks. Other factors were prolonged NICU stay ( $>2$ weeks), congenital heart disease
TABLE 1: Patient characteristics.

Gender

$\begin{array}{cc}\text { Male } & 48 \\ \text { Female } & 137\end{array}$

Birth history

Premature 45

Twin or triplet $\quad 14$

NICU admission

Median NICU length of stay (days) 12

Average NICU length of stay (days) 21

Location of $\mathrm{IH}$

$\begin{array}{cc}\text { Head and neck } & 130 \\ \text { Trunk } & 8 \\ \text { Extremity } & 15 \\ \text { Multifocal } & 31 \\ \text { Unknown } & 1\end{array}$

Comorbidities that may affect growth or development

$\begin{array}{cc}\text { Yes } & 43 \\ \text { No } & 142 \\ \text { Cardiac abnormalities } & 16 \\ \text { Laryngomalacia } & 5 \\ \text { Non-hemangioma surgery } & 23\end{array}$

Gestational history (mother)

$\begin{array}{cc}\text { Preeclampsia } & 23 \\ \text { Gestational diabetes } & 11 \\ \text { Placental abnormalities } & 12 \\ \text { First trimester bleeding } & 15 \\ \text { Trauma } & 23 \\ \text { Hypertension } & 23 \\ \text { Tobacco or alcohol } & 8 \\ \text { Amniocentesis } & 3 \\ \text { Chorionic villus sampling } & 0\end{array}$

Other medications

\begin{tabular}{cc} 
None & 53 \\
Antacid (PPI or H2 blocker) & 61 \\
Systemic steroid & 5 \\
Other & 59 \\
\hline
\end{tabular}

requiring surgery (excluding patent ductus arteriosus unless also coarctation of the aorta), congenital airway anomalies, adrenal insufficiency, hypothyroidism, and failure to thrive. Diseases such as asthma, eczema, autism, ADHD, and isolated low birth weight were not considered significant to growth and development. Patients presented to our outpatient vascular anomalies clinic for follow-up after initiation of propranolol. The mean follow-up time was 7.2 months. The weight-for-age, BMI-for-age, and weight-to-length $z$ -scores were calculated and compared to normative World Health Organization (WHO) values for pediatric growth. A mixed effects model was created with an unstructured covariance matrix to analyze weight, weight-for-age, weight-forlength, and BMI-for-age $z$-scores and percentiles. Random 

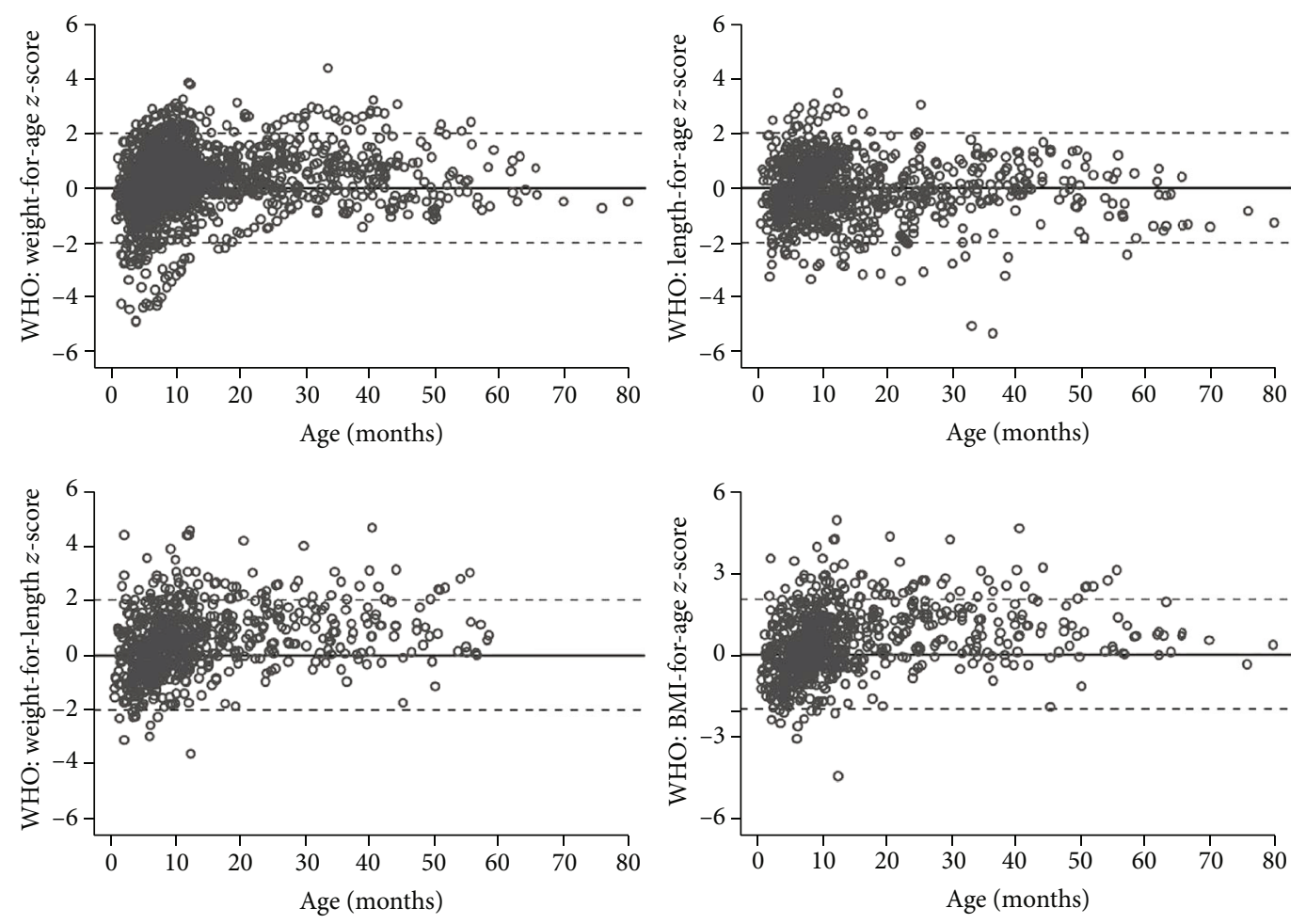

FIGURE 1: Treated and nontreated patients' $z$-scores compared to WHO standards.

intercepts and random slopes were evaluated by testing nested models using likelihood ratio tests.

In order to determine age of milestone achievement, side effects, efficacy, other treatments, and satisfaction with the therapy up to 48 months posttreatment, guardians were surveyed retrospectively, and additional chart results were tabulated. In a representative cohort, serum from age-matched children with IH treated and untreated with propranolol was collected for the determination of human growth hormone (hGH) levels during the course of their treatment.

\section{Results}

One hundred and eighty-five patients were included in the study, 48 males and 137 females (Table 1). Multiple gestation (twins and triplets) was found in $7.6 \%$ of the population. Eighty-eight patients had heights and weights while 97 patients had weight alone. Questionnaires regarding milestones, efficacy, and guardian satisfaction were performed on 174 patients, and a combination of both chart results and phone conducted surveys were tabulated. Thirty-eight patients had hGH measured; 11 of whom were on propranolol with the remaining untreated and healthy age matched. Serum measurements were drawn only on patients in the cohort undergoing anesthesia for related or unrelated procedures. Blood draws were considered too invasive in the clinic setting for this study.

There were 142 patients without and 43 patients with comorbidities that affect growth and development (Table 1). Birth weight in the patients was significantly lower in the cohort with comorbidities $(p<0.0001)$ suggesting patients were appropriately chosen for the cohort without

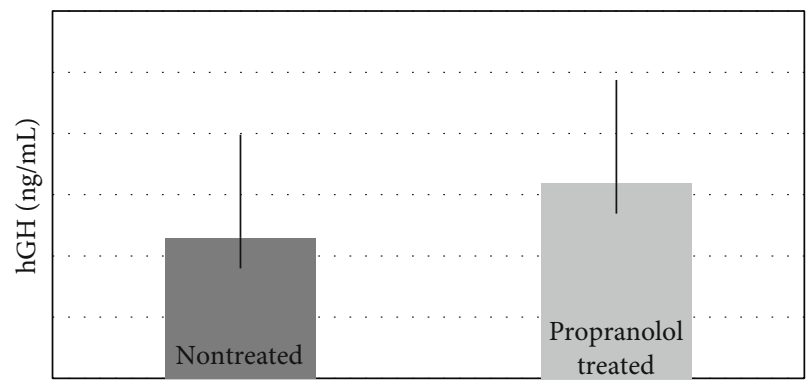

FIgURE 2: Mean hGH level in propranolol-treated $(6.38 \mathrm{ng} / \mathrm{mL})$ versus non-treated hemangioma patients $(4.59 \mathrm{ng} / \mathrm{mL})$. $\mathrm{SD}=3.15 \mathrm{ng} / \mathrm{mL}, p=0.16$.

comorbidities. Laryngomalacia was reported in 5 patients, and cardiac anomalies were reported in 16 patients. Twenty-three patients had general anesthesia for surgery for something other than $\mathrm{IH}$.

One patient whose guardian completed the questionnaire was excluded in the developmental portion due to global developmental delay, and another questionnaire was stopped short due to a cerebrovascular injury. If a parent could not recall the age at which milestones were achieved, that portion of the questionnaire was excluded. Families may not have responded to all the questions in the survey. Results reflect the total number of respondents for each question.

Of note, thirty-two (17.3\%) patients required a postnatal NICU admission unrelated to IH therapy with a length of staying ranging from 2 to 98 days and a median of 12 days. Fifty-eight were on medications other than propranolol. The most common unrelated pediatric condition was 
TABLE 2: Guardian reported side effects.

\begin{tabular}{lc}
\hline Side effect & Patients (\% of total) \\
\hline None & $115(62)$ \\
Reflux & $27(15)$ \\
Spit up more & $19(10)$ \\
Respiratory problems & $13(7)$ \\
Lethargy & $9(5)$ \\
Sleep disturbance & $8(4)$ \\
Mood alteration & $6(3)$ \\
Sweating & $2(1)$ \\
"Sickly" & $2(1)$ \\
Hyperactivity & $2(1)$ \\
Polyuria & $1(<1)$ \\
Allergy & $1(<1)$ \\
Gastritis & $1(<1)$ \\
\hline
\end{tabular}

gastroesophageal reflux, and accordingly, the most common other medication was a reflux medication such as an $\mathrm{H} 2$ blocker or a proton pump inhibitor (62 patients).

3.1. Anthropomorphic Measurements. The age-matched control heights and weights were compared in the propranolol treated and nontreated groups. The anthropomorphic data show patients in both cohorts were within or above the WHO normative values (Figure 1 and Supplementary Table 1). Some patients in our study population have higher weight-for-length and higher BMI-for-age than the WHO normative population. This observation seems to correlate with time on propranolol therapy (Supplementary Table 2). hGH levels were $6.38 \mathrm{ng} / \mathrm{mL}$ for propranolol-treated patients compared to $4.59 \mathrm{ng} / \mathrm{ml}$ in nontreated patients (Figure 2). This represents a standard deviation of $3.15 \mathrm{ng} / \mathrm{ml}$ and a $p$ value of 0.16 .

3.2. Side Effects. Twelve side effects from propranolol were reported in $70 / 185(37.8 \%)$ patients but rarely led to drug cessation (Table 2). The most common side effect was reflux, found in 27 (15\%) patients. Of note, 44 patients had symptomatic reflux before starting propranolol therapy, and 12 different questionnaire respondents reported it as a side effect. Other side effects included respiratory disturbances $(n=13)$, lethargy $(n=9)$, and sleep disturbance $(n=8)$.

3.3. Patient Satisfaction and Efficacy. Most guardian respondents reported that they were happy with the propranolol therapy 88/114 (77\%); 16/114 (14\%) were neutral, and 10/114 (9\%) were unhappy. 152 families reported clinical (80\%) improvement in color, size, or both. 107/154 (69.5\%) noticed a color change and $152 / 174(87.3 \%)$ reported a decrease in size of the $\mathrm{IH}$, most within one month of starting therapy $(60 / 89,67.4 \%)$. Twelve percent of patients had rebound after propranolol was stopped, and $73.9 \%$ of patients had a second type of treatment (laser, surgery, or injection) (Table 3). Ninety-three (53.4\%) patients had laser therapy in addition to the propranolol.
TABLE 3: Efficacy, side effects, and adjuvant therapy of propranolol based on guardian survey. Guardians may not have responded to each survey question thus creating variability in the number of respondents per question.

\begin{tabular}{|c|c|c|}
\hline \multicolumn{3}{|l|}{ Efficacy } \\
\hline Did not notice improvement & & 10 \\
\hline \multirow[t]{5}{*}{ Time to notice an improvement } & Less than one week & 34 \\
\hline & Less than one month & 32 \\
\hline & 1-2 months & 16 \\
\hline & More than 2 months & 13 \\
\hline & & 95 \\
\hline \multicolumn{3}{|l|}{ Color change } \\
\hline \multirow[t]{2}{*}{160} & Yes & $121(76)$ \\
\hline & No & $39(24)$ \\
\hline \multicolumn{3}{|l|}{ Decreased size } \\
\hline & Yes & $158(88)$ \\
\hline 180 & No & $22(22)$ \\
\hline \multicolumn{3}{|l|}{ Overall satisfaction } \\
\hline \multirow[t]{3}{*}{120} & Happy & $94(78)$ \\
\hline & Neutral & $16(13)$ \\
\hline & Unhappy & $10(8)$ \\
\hline \multicolumn{3}{|l|}{ Side effects } \\
\hline \multirow[t]{2}{*}{180} & Yes & $62(34)$ \\
\hline & No & $118(66)$ \\
\hline \multicolumn{3}{|l|}{ Recurrence/rebound } \\
\hline \multirow[t]{2}{*}{127} & Yes & $17(13)$ \\
\hline & No & $110(87)$ \\
\hline \multicolumn{3}{|l|}{ Adjuvant treatments* } \\
\hline \multirow[t]{5}{*}{180} & Laser & $97(54)$ \\
\hline & Surgery & $73(41)$ \\
\hline & Steroid & $35(19)$ \\
\hline & Scar revision & $1(<1)$ \\
\hline & Vincristine & $3(1)$ \\
\hline
\end{tabular}

${ }^{*}$ Note: some patients had multiple treatments.

3.4. Milestones. Based on the questionnaire respondents and chart results, patients met gross and fine motor milestones at or before the expected target age (Figure 3). This is true in both cohorts with or without comorbidities. Furthermore, by 4 years of age (Figure 4 ), less than $20 \%$ had not reached their expected milestone. Milestone achievement was not impacted by the presence of comorbidities that affect growth and development (Table 3).

\section{Discussion}

Propranolol has been in use for the treatment of infantile hemangiomas since 2008 and is well tolerated as evidenced in the present study. Although 12 different side effects were reported, some may have been observations and not directly 
Gross motor skills

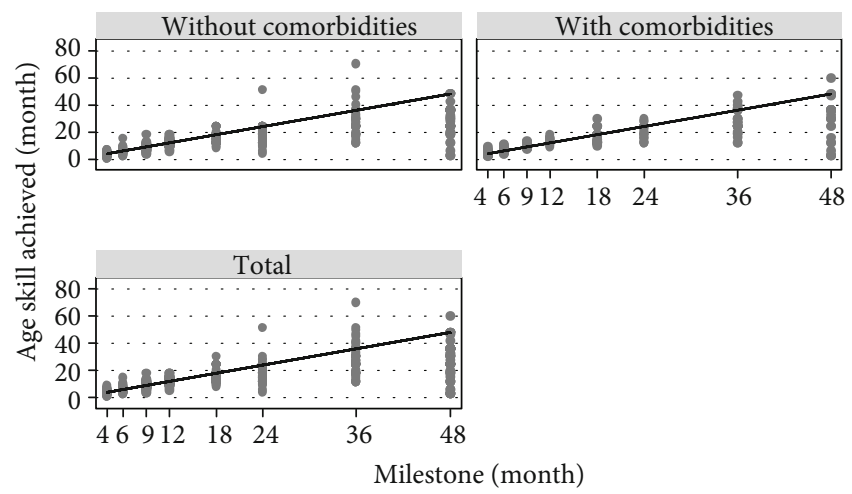

(a) Gross motor skills
Fine motor skills

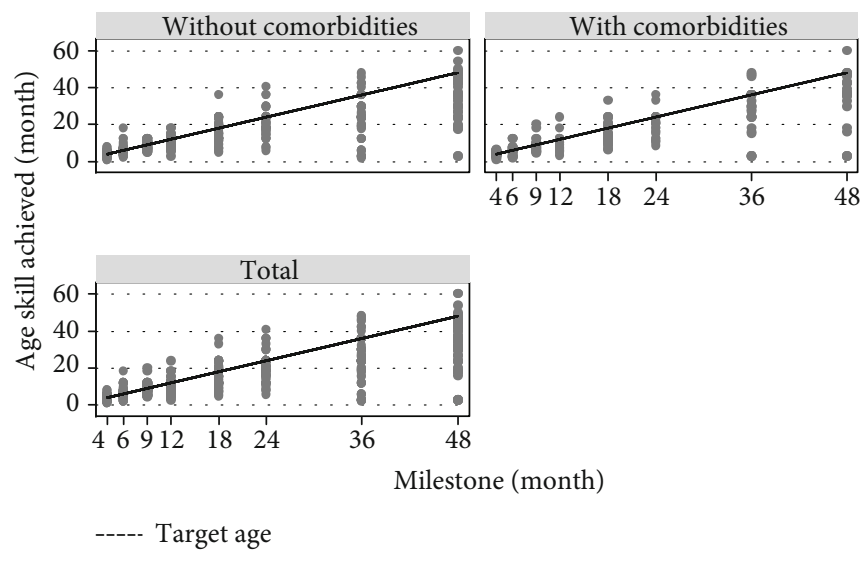

Patients (points) below target-age-line achieved milestone early

(b) Fine motor skills

Linear representation of fine and gross motor skill achievement
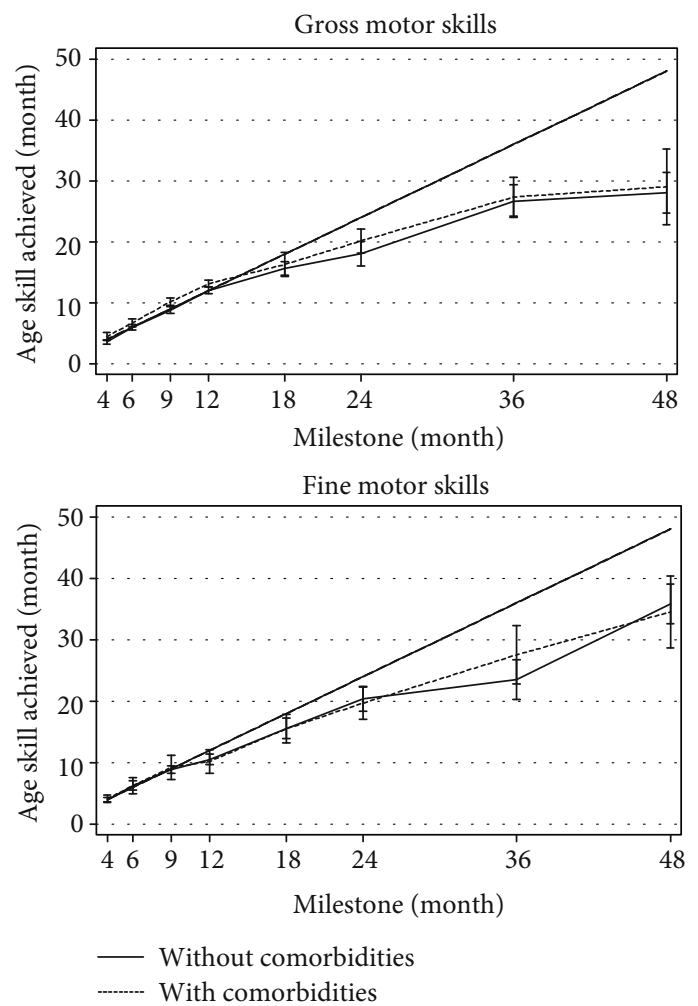

(c) Linear representation of fine and gross motor skill achievement

FIGURE 3: Patients treated with propranolol compared to expected developmental milestone. Each dot represents a patient at a moment in time. Patients below the target line reached the milestone before the target age.

related to propranolol use. Fifteen percent of our population reported reflux as a side effect. Although "spitting up" and "reflux" are difficult to distinguish, $10 \%$ of guardians thought that their children spit up more on propranolol.

In addition to being well tolerated, propranolol is known to be a successful treatment for infantile hemangiomas. In our study, most respondents were happy with the therapeutic effect. However, almost $74 \%$ of our population received secondary treatment, with the majority (53.4\%) of those receiving laser therapy. Laser therapy was performed to control symptoms such as bleeding or as a cosmetic adjunct for remaining tissue rather than for a propranolol treatment failure. A flash pump dye laser at $595 \mathrm{~nm}$ was used for this purpose. In most cases that required surgical excision, propranolol was used preoperatively, and surgery was then performed to remove remaining anetoderma or scar revision. All indices of efficacy, satisfaction, and side effects were not different between the cohorts with and without comorbidities.

Though there is not a single human study showing developmental delay or growth retardation due to propranolol, 
Milestone at 4 months
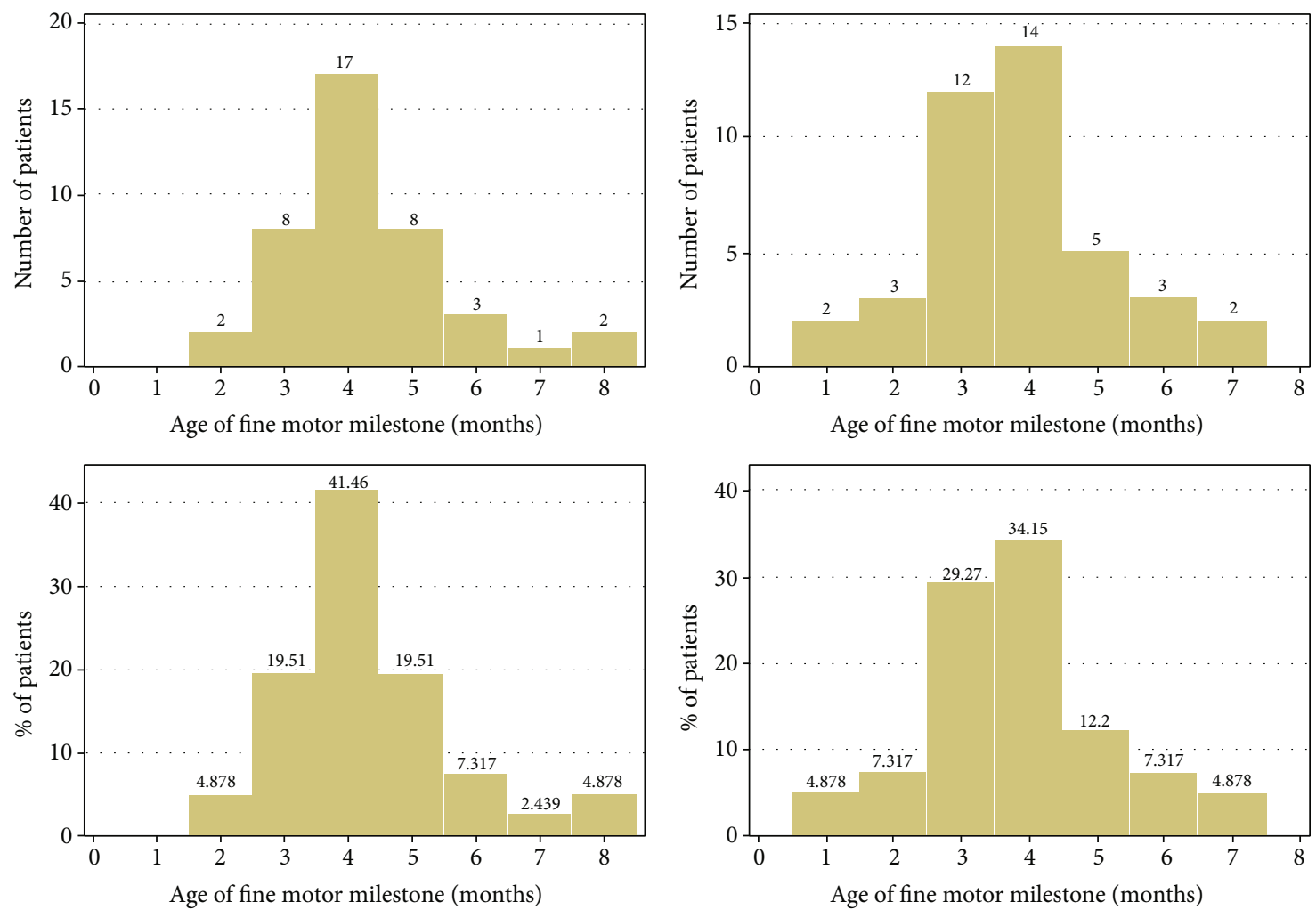

FIGURE 4: Patients treated with propranolol compared to expected developmental milestone. Number of patients per age that achieved 4month milestone and percentage of patients per age that achieved 4-month milestone.

concern might have been derived from previous studies showing intrauterine growth restriction (IUGR) and decreased perinatal growth in rats who were born to dams on beta-blockers $[23,24]$. Another animal study showed propranolol caused body weight deficits up to $33 \%$ [25]. Of note, this was at a dose of $75 \mathrm{mg} / \mathrm{kg}$ lavaged into suckling rats. Based on consensus data propranolol dose used to treat $\mathrm{IH}$ is recommended to be $2-3 \mathrm{mg} / \mathrm{kg} / \mathrm{day}$ [2]. Thus the lower dose of propranolol given to humans should not pose the same threat as the high dose used in rat studies. In fact, recent work by Moyakine et al. demonstrated no impact of low dose propranolol on human growth and development up to 4 years of age in infants treated for IH. The present findings refute prior animal investigations and corroborates prior human investigations.

Contrary to the concerns about pediatric growth, Ghigo et al. demonstrated beta-adrenergic antagonism increases growth hormone releasing hormone-induced GH secretion [26]. There is no known correlation between IH and growth hormone except one case of GH deficiency in a patient with facial hemangioma in the context of PHACE syndrome [27]. Accordingly, measurement of growth hormone in this study was chosen as an added objective measurement of pediatric growth in addition to heights and weights with evidence of higher hGH levels in patients treated with propranolol. Similarly, Altin et al. demonstrated that the physical growth rate of patients with a mean age of 2.64 was not affected by propranolol [28].
Because propranolol has been shown to affect the consolidation of memory [29], a concern was expressed that this may affect cognitive development in children. However, a study including 36 children with infantile hemangiomas on oral propranolol showed no effect on neurodevelopmental outcomes or CNS development [30], as well as another study in propranolol-treated children aged five to seven-and-a-half years old that showed no significant evidence of intelligence or memory development disturbance [31].

Our data suggest propranolol is a well-tolerated, effective treatment that does not impair normal growth and development. Although great lengths were taken to separate patients into two cohorts (with and without comorbidities) to better assess whether delay in growth or development was due to those factors, no appreciable difference was noted between the groups. Remarkably, without dividing the participants into two cohorts, there was not a statistically significant difference in milestones or growth at four years of age. This suggests these patients "catch up" developmentally. Explanations and validation of the ability to "catch up" in the face of seemingly growth-affecting comorbidities is beyond the scope of this paper.

Our data show hGH levels are not affected by propranolol therapy, but there are many factors other than hGH involved in pediatric growth that were not measured. Most children undergoing systemic propranolol treatment for IH did not have growth alterations. We were surprised to find some patients treated with propranolol had a higher 
weight-for-length BMI compared to WHO normative data. Although lengths and weights were within the $95^{\text {th }}$ percentile, the average BMI was higher. The initial concern of growth impairment is diminished; however, this may introduce a paradoxical concern of abnormal weight gain.

Care must be taken not to interpret this observation as a direct result of propranolol therapy. There is a population bias as this is a single-center study, and there is no control group of patients with hemangiomas not receiving therapy with whom to compare. There may also be a selection bias in the population of patients that presented to the center. These issues warrant further investigation. There are reports of patients taking propranolol for migraine that have experienced weight gain $[32,33]$. However, this issue has not been studied in the pediatric population except for one study showing weight gain in a patient on prolonged propranolol therapy for 6-12 months [34].

As propranolol is a relatively new therapy for infantile hemangiomas, long-term studies are still ongoing to completely evaluate the effects of propranolol in the pediatric population. This study does not evaluate other reported side effects of propranolol such as hypoglycemia, insomnia, and bradycardia; therefore, it is not a comprehensive study of propranolol safety. Furthermore, the primary aim of this study was not to examine the efficacy of propranolol for hemangiomas, which have been previously shown in other studies $[3,9,35]$ but instead to report the findings of the relationship between propranolol use, growth, and development.

Because therapy is effective and complete for most patients by one year of age, most patients do not follow in our clinic after the involution of the lesion for the 12 to 48 month period of milestone assessment. Thus, the questionnaire regarding milestone achievement after age one was completed by phone retrospectively. This methodology introduced recall bias.

\section{Conclusion}

We conclude that propranolol does not cause growth or developmental delay. This supports existing literature showing its safety in pediatric patients for the treatment of infantile hemangioma. Further study is warranted to evaluate the role of propranolol in the increase in weight-for-age and weight-for-length.

\section{Data Availability}

Data is available upon request. Please contact gtrichter@uams.edu if more information is required.

\section{Conflicts of Interest}

The authors declare that they have no conflicts of interest.

\section{Acknowledgments}

The authors would like to thank the following individuals for their contributions to this body of work.

\section{Supplementary Materials}

Supplementary tables were included to provide the reader with further information regarding the number of children above and below WHO $z$-scores, as well as the relationship between anthropometric measurements and the length of propranolol therapy. (Supplementary Materials)

\section{References}

[1] D. Krowchuk, I. Frieden, A. Mancini et al., "Clinical practice guideline for the management of Infantile Hemangiomas," Pediatrics, vol. 143, no. 1, article e20183475, 2019.

[2] B. A. Drolet, P. C. Frommelt, S. L. Chamlin et al., "Initiation and use of propranolol for infantile hemangioma: report of a consensus conference," Pediatrics, vol. 131, no. 1, pp. 128140, 2013.

[3] C. Léauté-Labrèze, E. D. de la Roque, T. Hubiche, F. Boralevi, J. B. Thambo, and A. Taïeb, "Propranolol for severe hemangiomas of infancy," The New England Journal of Medicine, vol. 358, no. 24, pp. 2649-2651, 2008.

[4] R. Hagen, E. Ghareeb, O. Jalali, and Z. Zinn, "Infantile hemangiomas: what have we learned from propranolol?," Current Opinion in Pediatrics., vol. 30, no. 4, pp. 499-504, 2018.

[5] I. J. Frieden and B. A. Drolet, "Propranolol for infantile hemangiomas: promise, peril, pathogenesis," Pediatric Dermatology, vol. 26, no. 5, pp. 642-644, 2009.

[6] L. P. Lawley, E. Siegfried, and J. L. Todd, "Propranolol treatment for hemangioma of infancy: risks and recommendations," Pediatric Dermatology, vol. 26, no. 5, pp. 610-614, 2009.

[7] T.-L. Yang, P. McMahon, D. De León, and J. Treat, "Use of propranolol for treating hemangiomas in infants with previously diagnosed hypoglycemic conditions," Pediatric Dermatology, vol. 33, no. 6, pp. 381-384, 2016.

[8] J. N. Love, T. L. Litovitz, J. M. Howell, and C. Clancy, "Characterization of fatal beta blocker ingestion: a review of the American Association of Poison Control Centers data from 1985 to 1995," Journal of Toxicology. Clinical Toxicology, vol. 35, pp. 353-359, 2009.

[9] L. M. Buckmiller, P. D. Munson, U. Dyamenahalli, Y. Dai, and G. T. Richter, "Propranolol for infantile hemangiomas: early experience at a tertiary vascular anomalies center," Laryngoscope, vol. 120, no. 4, pp. 676-681, 2010.

[10] A. Georgountzou, E. Karavitakis, A. Klimentopoulou, A. Xaidara, and T. Kakourou, "Propranolol treatment for severe infantile hemangiomas: a single-centre 3-year experience," Acta Paediatrica, vol. 101, no. 10, pp. e469-e474, 2012.

[11] S. R. Parikh, D. H. Darrow, J. F. Grimmer, S. C. Manning, G. T. Richter, and J. A. Perkins, "Propranolol use for infantile hemangiomas," JAMA Otolaryngology. Head \& Neck Surgery, vol. 139, no. 2, pp. 153-156, 2013.

[12] G. T. Richter and A. B. Friedman, "Hemangiomas and vascular malformations: current theory and management," International Journal Of Pediatrics, vol. 2012, Article ID 645678, 10 pages, 2012.

[13] R. Sidbury, "Update on vascular tumors of infancy," Current Opinion in Pediatrics, vol. 22, no. 4, pp. 432-437, 2010.

[14] A. P. Zimmermann, S. Wiegand, J. A. Werner, and B. Eivazi, "Propranolol therapy for infantile haemangiomas: review of 
the literature," International Journal of Pediatric Otorhinolaryngology, vol. 74, no. 4, pp. 338-342, 2010.

[15] A. L. Marqueling, V. Oza, I. J. Frieden, and K. B. Puttgen, "Propranolol and infantile hemangiomas four years later: a systematic review," Pediatric Dermatology, vol. 30, no. 2, pp. 182-191, 2013.

[16] K. W. Rosbe, K. Y. Suh, A. K. Meyer, S. M. Maguiness, and I. J. Frieden, "Propranolol in the management of airway infantile hemangiomas," Archives of Otolaryngology - Head \& Neck Surgery, vol. 136, no. 7, pp. 658-665, 2010.

[17] A. Zvulunov, C. McCuaig, I. J. Frieden et al., "Oral propranolol therapy for infantile hemangiomas beyond the proliferation phase: a multicenter retrospective study," Pediatric Dermatology, vol. 28, no. 2, pp. 94-98, 2011.

[18] C. Leaute-Labreze, P. Hoeger, J. Mazereeuw-Hautier et al., “A randomized, controlled trial of oral propranolol in infantile hemangioma," New England Journal of Medicine, vol. 372, no. 8, pp. 735-746, 2015.

[19] A. Izadpanah, A. Izadpanah, J. Kanevsky, E. Belzile, and K. Schwarz, "Propranolol versus corticosteroids in the treatment of infantile hemangioma: a systematic review and meta-analysis," Plastic and Reconstructive Surgery, vol. 131, no. 3, pp. 601-613, 2013.

[20] A. Erbay, F. Sarialioglu, B. Malbora et al., "Propranolol for infantile hemangiomas: a preliminary report on efficacy and safety in very low birth weight infants," Turkish Journal of Pediatrics, vol. 52, no. 5, p. 450, 2010.

[21] M. G. Kumar, C. Coughlin, and S. J. Bayliss, "Outpatient use of oral propranolol and topical timolol for infantile hemangiomas: survey results and comparison with propranolol consensus statement guidelines," Pediatric Dermatology, vol. 32, no. 2, pp. 171-179, 2015.

[22] J. Ge, J. Zheng, L. Zhang, W. Yuan, and H. Zhao, "Oral propranolol combined with topical timolol for compound infantile hemangiomas: a retrospective study," Scientific Reports, vol. 6, no. 1, p. 19765, 2016.

[23] N. Schoenfeld, O. Epstein, L. Nemesh, M. Rosen, and A. Atsmon, "Effects of propranolol during pregnancy and development of rats. I. Adverse effects during pregnancy," Pediatric Research, vol. 12, no. 7, pp. 747-750, 1978.

[24] N. Schoenfeld, O. Epstein, M. Rosen, and A. Atsmon, "Effects of propranolol during pregnancy and development of rats. II. Adverse effects on development," European Journal of Pediatrics, vol. 143, no. 3, pp. 194-195, 1985.

[25] G. Redmond, "Propanolol inhibits brain and somatic growth in the rat," Nature, vol. 15, p. 645, 1981.

[26] A. V. Moyakine, J. M. Kerstjens, S. Spillekom-van Koulil, and C. J. M. van der Vleuten, "Propranolol treatment of infantile hemangioma ( $\mathrm{IH})$ is not associated with developmental risk or growth impairment at age 4 years," Journal of the American Academy of Dermatology, vol. 75, no. 1, pp. 59-63.e1, 2016.

[27] E. Ghigo, J. Bellone, E. Arvat et al., "Effects of alpha- and betaadrenergic agonists and antagonists on growth hormone secretion in man," Journal of Neuroendocrinology, vol. 2, no. 4, pp. 473-476, 1990.

[28] H. Altin, H. Alp, F. Şap, Z. Karataş, T. Baysal, and S. Karaaslan, "PHACE syndrome with growth hormone deficiency and absence of bilateral internal carotid arteries: a case report," Pediatric Dermatology, vol. 29, no. 3, pp. 316-319, 2012.

[29] L. Hu, B. Zhou, H. Huang et al., "Effects of systemic propranolol treatment on physical growth of patients with infantile hemangiomas," Journal of Dermatology, vol. 43, no. 10, pp. 1160-1166, 2016.

[30] M. H. Lonergan, L. A. Olivera-Figueroa, R. K. Pitman, and A. Brunet, "Propranolol's effects on the consolidation and reconsolidation of long-term emotional memory in healthy participants: a meta-analysis," Journal of Psychiatry \& Neuroscience, vol. 38, no. 4, pp. 222-231, 2013.

[31] C. Wang, W. Wang, B. Xiang, S. Chen, F. Xiong, and Y. Ji, "Effects of Propranolol on Neurodevelopmental Outcomes in Patients with Infantile Hemangioma: A Case-Control Study," BioMed Research International, vol. 2018, Article ID 5821369, 6 pages, 2018.

[32] L. Tang, J. W. Hing, J. Tang et al., "Predicting complications with pretreatment testing in infantile haemangioma treated with oral propranolol," British Journal of Ophthalmology, vol. 100, no. 7, pp. 902-906, 2016.

[33] F. R. Taylor, "Weight change associated with the use of migraine-preventive medications," Clinical Therapeutics, vol. 30, no. 6, pp. 1069-1080, 2008.

[34] I. Martinez-Mir, J. Navarro-Badenes, V. Palop, F. J. MoralesOlivas, and E. Rubio, "Weight gain induced by long-term propranolol treatment," The Annals of Pharmacotherapy, vol. 27, no. 4, p. 512, 1993.

[35] M. Oksiuta, E. Matuszczak, W. Dębek, E. Dzienis-Koronkiewicz, A. Hermanowicz, and M. Tylicka, "Treatment of problematic infantile hemangiomas with propranolol: a series of 40 cases and review of the literature," Postępy Higieny i Medycyny Doświadczalnej (Online), vol. 68, pp. 1138-1144, 2014. 